\title{
REMARKS ON MINIMAL IDENTITIES FOR ALGEBRAS
}

\author{
A. S. AMITSUR AND J. LEVITZKI
}

1. Introduction. The purpose of the present note is to supplement in some points the results obtained by the authors in a previous com. munication. Let $A_{n}$ denote the total matric algebra of order $n^{2}$ over a field $F$. In $[1]^{1}$ we have determined the totality of minimal identities satisfied by $A_{n}$, in all cases where $n>2$ or $F \neq P_{2}$, where $P_{2}$ denotes the prime field of characteristic 2 . In all these cases each minimal polynomial is (but for a numerical factor) either a standard polynomial of degree $2 n$ or a sum of such standard polynomials. This is not so if $n \leqq 2$ and $F=P_{2}$.

In $[1$, Theorem 6$]$ we have shown that in these two exceptional cases nonlinear minimal polynomials do exist. In $\$ 2$ of the present note we determine the totality of the minimal identities in these exceptional cases.

In [1] it was shown that all linear minimal polynomials of a simple or a semi-simple algebra are again the standard polynomials and their linear combinations. In $\S 3$ we prove that in general all minimal polynomials of a semi-simple algebra are linear, hence all results on minimal polynomials for total matric algebras, which we have obtained in [1], may be extended to simple and semi-simple algebras.

For an algebra $A$ with a radical $B$ we have found in [1] an identity whose degree depends on the index of $B$ and the orders of the simple constituents of the difference algebra $A-B$. This yields an upper bound and a lower bound for the degree of a minimal identity in the non-semi-simple case. In $\S 4$ of the present note we show by examples that these estimates are in a way the best possible ones.

2. The minimal polynomials in the exceptional cases. We first dispose of the case $n=1$ and $F=P_{2}$, that is, $A_{1}=P_{2}$. The only nonlinear minimal polynomial depending on one indeterminate $x$ is the polynomial $x^{2}+x$. The only minimal polynomial depending on two indeterminates $x_{1}, x_{2}$ and linear in each of these indeterminates is by $\left[1\right.$, Theorems 1, 7] the standard polynomial $S\left(x_{1}, x_{2}\right)=x_{1} x_{2}-x_{2} x_{1}$.

For an arbitrary set of indeterminates $x_{1}, \cdots, x_{k}(k \geqq 2)$, denote by $M_{1}$ the module over $P_{2}$ defined by the set of all minimal polynomials of $A_{1}$, depending on $x_{1}, \cdots, x_{k}$. It is readily seen that a basis of $M_{1}$ is constituted by the following polynomials,

Received by the editors January 11, 1950.

${ }^{1}$ Numbers in brackets refer to the bibliography at the end of the paper. 


$$
x_{i}^{2}+x_{i}, \quad S\left(x_{j_{1}}, x_{j_{2}}\right)
$$

where $i=1,2, \cdots, k$ and $\left(j_{1}, j_{2}\right)$ ranges over all combinations of two letters out of $k$. The dimensionality of this module is, therefore, $k+C_{k, 2}$.

Consider now the algebra $A_{2}$ over the field $F=P_{2}$, and let $f$ $=f\left(x_{1}, \cdots, x_{k}\right)$ be a minimal polynomial of $A_{2}$ (hence of degree 4) so that each monomial of $f$ has a degree $\geqq 1$ in each of the $x_{i}$, that is, $k \leqq 4$. It is sufficient to determine all minimal polynomials satisfying this condition, since by [1, Lemma 7] every minimal polynomial may be represented as a sum of minimal polynomials of this type.

The elements of $A_{2}$ over $P_{2}$ satisfy one of the following 4 equations:

$$
y^{2}=0, \quad y^{2}=y, \quad y^{2}=1, \quad y^{2}=y+1
$$

and each of these equations is satisfied by some elements of $A_{2}$. This implies that no identity of the form: $x^{4}+\beta_{1} x^{3}+\beta_{2} x^{2}+\beta_{3} x+\beta_{4}=0$ $\left(\beta_{i} \in P_{2}\right)$ is satisfied by $A_{2}$, and hence $k \geqq 2$. If further $k=4$, that is, $f$ is linear in each of the indeterminates, by [1, Theorem 2] we know that $f$ is the standard polynomial $S\left(x_{1}, x_{2}, x_{3}, x_{4}\right)$. Thus it remains to determine all minimal polynomials $f\left(x_{1}, \cdots, x_{k}\right)$ with $k=2,3$. form

Consider first the case $k=3$. In this case we may write $f$ in the

$$
f=f_{0}+f_{1}+f_{2}+f_{3}
$$

where each of the monomials of $f_{0}$ with a nonzero coefficient is of degree 1 in each $x_{j}$ (that is, $f_{0}$ is either zero or of degree 3 ) while for $i \geqq 1$, each monomial of $f_{i}$ with a nonzero coefficient has degree 2 in $x_{i}$ and degree 1 in $x_{k}, k \neq i$. This implies that at least one of the $f_{i}$, $i \geqq 1$, is not equal to 0 and we may assume that $f_{1} \neq 0$. Hence (1) is a special case of formula (30) in [1] and we may apply the results obtained in [1]. Thus we have according to formula (34) of [1]:

$$
f=f_{0}+\alpha_{1} p_{1}+\alpha_{2} p_{2}+\alpha_{3} p_{3}, \quad \alpha_{1} \neq 0,
$$

where $p_{i}$ is the sum of all 12 monomials having degree 2 in $x_{i}$ and degree 1 in $x_{k}, k \neq i$.

First apply the substitution $x_{1}=e_{12}, x_{2}=e_{22}, x_{3}=e_{21}$., The only monomial linear in each $x_{i}$ which yields under this substitution the unit $e_{11}$ is $x_{1} x_{2} x_{3}$. It is readily verified that $p_{i}\left(e_{12}, e_{22}, e_{21}\right)=0, i=1,3$, and $p_{2}\left(e_{12}, e_{22}, e_{21}\right)=e_{11}$. This implies that $\alpha_{2}$ is also the coefficient of the monomial $x_{1} x_{2} x_{3}$ of $f_{0}$. A permutation of $x_{1}$ and $x_{3}$ in the last substitution shows that $\alpha_{2}$ is also the coefficient of the monomial $x_{3} x_{2} x_{1}$. Similar results may be obtained for $\alpha_{1}$ and $\alpha_{3}$, hence 


$$
\begin{aligned}
f= & \alpha_{1}\left(x_{2} x_{1} x_{3}+x_{3} x_{1} x_{2}+p_{1}\right)+\alpha_{2}\left(x_{1} x_{2} x_{3}+x_{3} x_{2} x_{1}+p_{2}\right) \\
& +\alpha_{3}\left(x_{1} x_{3} x_{2}+x_{2} x_{3} x_{1}+p_{3}\right) .
\end{aligned}
$$

Now apply the substitution: $x_{1}=e_{11}, x_{2}=e_{12}, x_{3}=e_{22}$. The only monomials of $f$ which yield $e_{12}$ are $x_{1}^{2} x_{2} x_{3}, x_{1} x_{2} x_{3}$, and $x_{1} x_{2} x_{3}^{2}$, hence $\alpha_{1}+\alpha_{2}+\alpha_{3}=0$. Since each $\alpha_{i}$ is either 0 or 1 , and $\alpha_{1} \neq 0$, it follows that either $\alpha_{2}=0, \alpha_{3}=1$, or $\alpha_{2}=1, \alpha_{3}=0$. Denote by $(i, j, k)$ any permutation of the three indices $1,2,3$ and put

$$
G_{i}=x_{j} x_{i} x_{k}+x_{k} x_{i} x_{j}+p_{i}, \quad i=1,2,3 ;
$$

then $f$ is either $G_{1}+G_{2}$ or $G_{1}+G_{3}$. Consider the three polynomials

$$
H_{1}=G_{2}+G_{3}, \quad H_{2}=G_{1}+G_{3}, \quad H_{3}=G_{1}+G_{2} .
$$

Each polynomial $H_{i}$ is of degree 1 in $x_{i}$ and of degree 2 in $x_{k}, k \neq i$. Since the underlying field is of characteristic 2 it follows that $H_{1}+H_{2}$ $+H_{3}=0$. The polynomials $H_{i}$ may be transformed into each other by changing the roles of the indeterminates, and thus it follows that $f$ must be one of the three polynomials $H_{1}, H_{2}, H_{3}$.

Since it was shown in [1, Theorem 6] that the identity

$$
Q(x, y)=x y^{3}+y x y^{2}+y^{2} x y+y^{3} x+x y^{2}+y^{2} x=0
$$

is satisfied by $A_{2}$ over $P_{2}$, and it is readily seen that

$$
Q\left(x_{1}, x_{2}+x_{3}\right)-Q\left(x_{1}, x_{2}\right)-Q\left(x_{1}, x_{3}\right)=H_{1}\left(x_{1}, x_{2}, x_{3}\right),
$$

we conclude that the identity $H_{1}=0$ and hence, also, $H_{2}=0$ and $H_{3}=0$ are indeed satisfied by the algebra $A_{2}$ over $P_{2}$.

Thus we have:

THEOREM 1a. The polynomial $f\left(x_{1}, x_{2}, x_{3}\right)$, where each term is of degree $\geqq 1$ in each $x_{i}$, is a minimal polynomial of $A_{2}$ over $P_{2}$ if and only if $f$ is one of the polynomials $H_{1}, H_{2}, H_{3}$.

We now turn to the case where the minimal polynomial $f\left(y_{1}, y_{2}\right)$ depends on 2 indeterminates. The polynomial $f$ has monomials with degree $\geqq 2$ in one of the $y$ 's, say in $y_{2}$. The polynomial $F_{1}\left(x_{1}, x_{2}, x_{3}\right)$ defined by

$$
F_{1}\left(x_{1}, x_{2}, x_{3}\right)=f\left(x_{1}, x_{2}+x_{3}\right)-f\left(x_{1}, x_{2}\right)-f\left(x_{1}, x_{3}\right)
$$

is again a minimal polynomial of $A_{2}$. It is evident that $F_{1}$ is symmetric in $x_{2}$ and $x_{3}$, and each term of $F_{1}$ is of degree $\geqq 1$ in each of the $x$ 's. Hence it follows by the preceding theorem that. $F_{1}\left(x_{1}, x_{2}, x_{3}\right)$ $\equiv H_{1}\left(x_{1}, x_{2}, x_{3}\right)$. We have already seen that

$$
Q_{1}\left(x_{1}, x_{2}+x_{3}\right)-Q_{1}\left(x_{1}, x_{2}\right)-Q_{1}\left(x_{1}, x_{3}\right)=H_{1}\left(x_{1}, x_{2}, x_{3}\right)
$$


where $Q_{1}\left(y_{1}, y_{2}\right)=y_{1} y_{2}^{3}+y_{2} y_{1} y_{2}^{2}+y_{2}^{2} y_{1} y_{2}+y_{2}^{3} y_{1}+y_{1} y_{2}^{2}+y_{2}^{2} y_{1}$. Hence, by putting $f_{1}\left(y_{1}, y_{2}\right)=f\left(y_{1}, y_{2}\right)-Q_{1}\left(y_{1}, y_{2}\right)$, it follows that $f_{1}\left(x_{1}, x_{2}+x_{3}\right)$ $-f_{1}\left(x_{1}, x_{2}\right)-f_{1}\left(x_{1}, x_{3}\right) \equiv 0$ identically in $x_{1}, x_{2}, x_{3}$. This implies that either $f_{1}\left(y_{1}, y_{2}\right) \equiv 0$ or $f_{1}\left(y_{1}, y_{2}\right)$ is linear in $y_{2}$. In the former case we obtain $f\left(y_{1}, y_{2}\right) \equiv Q_{1}\left(y_{1}, y_{2}\right)$, while in case $f_{1}\left(y_{1}, y_{2}\right) \not \equiv 0$ we know that $f_{1}\left(y_{1}, y_{2}\right)$ is again a minimal polynomial of $A_{2}$ over $P_{2}$ such that each term of $f_{1}$ is of degree $\geqq 1$ in $y_{1}$ and in $y_{2}$. Since $f_{1}$ is linear in $y_{2}$, it must be of degree $\geqq 2$ in $y_{1}$. Hence, in a similar manner we show that the polynomial $F_{2}\left(x_{1}, x_{2}, x_{3}\right)=f_{1}\left(x_{1}+x_{2}, x_{3}\right)-f_{1}\left(x_{1}, x_{3}\right)-f_{1}\left(x_{2}, x_{3}\right)$ is equal to $H_{3}$. Since the polynomial

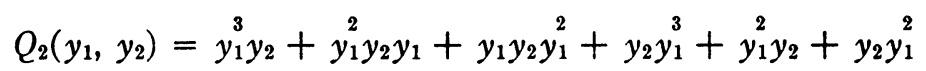

also satisfies $Q_{2}\left(x_{1}+x_{2}, x_{3}\right)-Q_{2}\left(x_{1}, x_{3}\right)-Q_{2}\left(x_{2}, x_{3}\right)=H_{3}\left(x_{1}, x_{2}, x_{3}\right)$, it follows similarly that either the polynomial $f_{1}\left(y_{1}, y_{2}\right)-Q_{2}\left(y_{1}, y_{2}\right)$ $=f_{2}\left(y_{1}, y_{2}\right)$ is zero, or $f_{2}$ must be linear in $y_{1}$. The latter possibility leads to a contradiction, since in this case $f_{2}$ must be linear in $y_{2}$ also, which implies that the general degree of $f_{2}$ is less than 4 , which is impossible, since $f_{2}$ is a minimal polynomial of $A_{2}$. This implies that $f\left(y_{1}, y_{2}\right)=Q_{1}\left(y_{1}, y_{2}\right)+Q_{2}\left(y_{1}, y_{2}\right)$. It has already been shown that the identities $Q_{1}\left(y_{1}, y_{2}\right)=0, Q_{2}\left(y_{1}, y_{2}\right)=0$ hold in $A_{2}$ over $P_{2}$. Hence, also the identity $Q_{1}+Q_{2}=0$ holds in $A_{2}$ over $P_{2}$ and we have:

Theorem 1b. A polynomial $f\left(y_{1}, y_{2}\right)$, such that each term of $f$ is of degree not less than 1 in $y_{1}$ and $y_{2}$, is a minimal polynomial of $A_{2}$ over $P_{2}$ if and only if $f=Q_{1}$, or $f=Q_{2}$, or $f=Q_{1}+Q_{2}$.

By summarizing above results we get:

Theorem 1. Let $f\left(x_{1}, \cdots, x_{k}\right)$ be a minimal polynomial of $A_{2}$ over $P_{2}$, such that each monomial of $f$ is of a degree $\geqq 1$ in each $x_{i}$, then $2 \leqq k \leqq 4$, and:

(1) If $k=4$ then $f\left(x_{1}, x_{2}, x_{3}, x_{4}\right)=S\left(x_{1}, x_{2}, x_{3}, x_{4}\right)$.

(2) If $k=3$ then $f$ is one of the polynomials $H_{1}, H_{2}, H_{3}$.

(3) If $k=2$ then $f$ is one of the polynomials $Q_{1}, Q_{2}, Q_{1}+Q_{2}$.

Since by [1, Lemma 7] it follows that every minimal polynomial may be represented as a sum of polynomials of the type mentioned in the preceding theorem, we have:

TheOREM 2. The module $M_{2}$ defined by the minimal polynomials of $A_{2}$ over $P_{2}$, depending on the indeterminates $x_{1}, x_{2}, \cdots, x_{k}, k \geqq 4$, has the dimensionality $C_{k, 4}+2 C_{k, 3}+2 C_{k, 2}$. As a basis for $M_{2}$ we may choose the polynomials:

$S\left(x_{i_{1}}, x_{i_{2}}, x_{i_{3}}, x_{i_{4}}\right), H_{1}\left(x_{i_{1}}, x_{i_{9}}, x_{i_{3}}\right), H_{2}\left(x_{i_{1}}, x_{i_{2}}, x_{i_{3}}\right), Q_{1}\left(x_{i_{1}}, x_{i_{2}}\right), Q_{2}\left(x_{i_{1}}, x_{i_{2}}\right)$ 
where $\left(i_{1}, i_{2}, i_{3}, i_{4}\right)$ is an arbitrary combination of 4 letters out of $k$.

For $k=3$ we have the basis

$$
H_{1}\left(x_{1}, x_{2}, x_{3}\right), \quad H_{2}\left(x_{1}, x_{2}, x_{3}\right), \quad Q_{1}\left(x_{i_{1}}, x_{i_{3}}\right), \quad Q\left(x_{i_{1}}, x_{i_{2}}\right)
$$

where $\left(i_{1}, i_{2}\right)$ is an arbitrary combination of 2 letters out of 3 , and in this case the dimensionality of $M_{2}$ is 8 .

For $k=2$, the dimensionality of $M_{2}$ is 2 and we have the basis

$$
Q_{1}\left(x_{1}, x_{2}\right), \quad Q_{2}\left(x_{1}, x_{2}\right) .
$$

For later reference we need the following remark.

REMARK. If in $H_{1}$ (resp. $Q_{1}$ ) one ignores the order of the factors, one obtains $H_{1}=4 x_{1} x_{2} x_{3}+12 x_{1} x_{2}^{2} x_{3}+12 x_{1} x_{2} x_{3}^{2}$ (resp. $Q_{1}=2 x_{1} x_{2}^{2}+4 x_{1} x_{2}^{3}$ ).

3. Simple and semi-simple algebras. We shall need the following generalization of Kaplansky's Lemma 3 [2].

Lemma. If an algebra $A$ over $F$ satisfies an identity $f\left(x_{1}, \cdots, x_{k}\right)=0$ which is homogeneous in each $x_{i}$ and of degree not greater than 2 in each $x_{i}$, then the given identity is satisfied also by the direct product $A \times G$, where $G$ is a field containing $F$.

Proof. We prove the lemma by induction on the number of the indeterminates $x_{i}$ whose degree in $f$ is 2 .

By Lemma 3 in [2] our lemma holds when $f$ is linear in each $x_{i}$. Suppose now that $f\left(x_{1}, \cdots, x_{k}\right)$ is of degree 2 in $x_{i}$ where $1 \leqq i \leqq k$ and consider the polynomial

$$
\begin{aligned}
(3)= & f\left(x_{1}, \cdots, x_{i-1}, u+v, x_{i+1}, \cdots\right)-f\left(\cdots, x_{i-1}, u, x_{i+1}, \cdots\right) \\
& -f\left(\cdots, x_{i-1}, v, x_{i+1}, \cdots\right) .
\end{aligned}
$$

This polynomial is apparently homogeneous in each of its indeterminates, and the number of indeterminates whose degree in $g_{i}$ is 2 is less than that of $f$. Since the identity $g_{i}=0$ holds in $A$, we may assume (by induction) that the identity $g_{i}=0$ holds also in $A \times G$. By (3) we have, for any sequence of $k+1$ elements $b_{1}, b_{2}, a_{1}, \cdots, a_{i-1}$, $a_{i+1}, \cdots, a_{k}$ belonging to $A \times G$, the relation

$$
\begin{aligned}
f\left(a_{1}, \cdots, a_{i-1}, b_{1}+b_{2}, a_{i+1}, \cdots, a_{k}\right) & \\
& =f\left(\cdots, b_{1}, \cdots\right)+f\left(\cdots, b_{2}, \cdots\right) .
\end{aligned}
$$

Since relation (4) evidently holds also in case $f\left(x_{1}, \cdots, x_{k}\right)$ is linear in $x_{i}$, we may assume its validity for each $x_{i}, 1 \leqq i \leqq k$. Now each element $a \in A \times G$ has the form $a=\sum \gamma_{j} a_{j}$ where $a_{j} \in A$ and $\gamma_{j} \in G$. Hence in view of (4) it remains only to show that $f\left(x_{1}, \cdots, x_{k}\right)=0$ 
for $x_{i}=\delta_{i} b_{i}, b_{i} \in A, \delta_{i} \in G$. This is evident, since $f\left(\delta_{1} b_{1}, \cdots, \delta_{k} b_{k}\right)$ $=\delta_{1}^{\nu_{1}} \cdots \delta_{k}^{\nu_{k}} f\left(b_{1}, \cdots, b_{k}\right)=0$ where $\nu_{i}$ is the degree of $x_{i}$ in $f$. This completes the proof of the lemma.

With the aid of the preceding lemma we now extend Theorems 4 and 5 of [1] to the general case of simple algebras by proving the following theorem:

THEOREM 3. Let $A$ be a simple algebra of order $n^{2}$ over its centre, and suppose that $A$ is neither $P_{2}$ nor is $A$ the total matric algebra $A_{2}$ over $P_{2}$. Then $f\left(x_{1}, \cdots, x_{m}\right)=0$ is a minimal identity of $A$ if and only if $m$ $\geqq 2 n$ and

$$
f\left(x_{1}, \cdots, x_{m}\right)=\sum_{(i)} \alpha_{(i)} S\left(x_{i_{1}}, \cdots, x_{i_{2 n}}\right)
$$

where the sum ranges over all $C_{m, 2 n}$ combinations (i) of $2 n$ letters out of $m$ letters, and the $\alpha_{(i)}$ are in the underlying field.

Proof. Since $A$ is a normal simple algebra over its centre $C$, there exists a field $G$ containing $C$, for which $A \times G$ over $C$ is the total matric algebra $A_{n}$ over $G$. The algebra $A \times G$ is apparently neither the algebra $A_{2}$ over $P_{2}$ nor is $A \times G$ the field $P_{2}$ since $A$ is not one of these exceptional cases. We assert first that the minimal polynomials of $A$ are linear in all their indeterminates. Indeed suppose that $A$ possesses nonlinear minimal polynomials. Then by Lemmas 5 and 6 of [1] we may assume that there exists a minimal polynomial $f\left(x_{1}, \cdots, x_{m}\right)$ of $A$ which is homogeneous and of degree not greater than 2 in each of the $x$ 's and of degree 2 in some of them. By the preceding lemma it follows that the identity $f=0$ is satisfied also by the algebra $A \times G$. But since $A \times G$ is a total matric algebra and $A \times G$ is not one of the exceptional cases, this contradicts Theorem 5 of [1]. This implies that every minimal identity $f=0$ satisfied by $A$ is a linear identity. By Theorem 4 of [1] it follows, therefore, that $m \geqq 2 n$, and

$$
f\left(x_{1}, \cdots, x_{m}\right)=\sum_{(i)} \alpha_{(i)} S\left(x_{i_{1}}, \cdots, x_{i_{z_{n}}}\right)
$$

where $\alpha_{(i)} \in G$. One readily verifies that $\alpha_{(i)} \in F$, since $f$ is a polynomial in $F$.

By [1, Theorem 7] we know that the converse is true also, that is, the identities of the type $\sum_{(i)} \alpha_{(i)} S\left(x_{i_{1}}, \cdots, x_{i_{2 n}}\right)=0$ are satisfied by the algebra $A$. This completes the proof of the theorem.

Consider now a semi-simple algebra $A$ over $F$, and suppose that $A$ is a direct sum of the simple algebras $A^{\prime}, A^{\prime \prime}, \cdots, A^{(k)}$. Denote by $n_{\mathbf{i}}^{2}$ the order of $A^{(i)}$ over its centre, and put $n^{2}=\max \left(n_{1}^{2}, \cdots, n_{\mathbf{k}}^{2}\right)$. 
It is readily verified ${ }^{2}$ that an identity $f=0$ is satisfied by $A$ if and only if it is satisfied by every constituent $A^{(i)}$.

Consider first the following exceptional cases:

(I) All algebras $A^{(i)}$ are isomorphic with $P_{2}$. This implies $n=1$ and $F=P_{2}$. Hence, $f=0$ is a minimal identity of $A$ if and only if $f=0$ is a minimal identity of $P_{2}$, and these identities were determined in the previous section.

(II) Some algebras $A^{(i)}$ are the algebras $A_{2}$ over $P_{2}$, and the remaining algebras $A^{(i)}$ (if any) are commutative fields of characteristic 2. This implies $F=P_{2}, n=2$.

By the remark at the end of the previous section it follows that all minimal identities of $A_{2}$ over $P_{2}$ are satisfied also by commutative fields of characteristic 2 . This implies that in case (II) the module of the minimal polynomials of the algebra $A$ is the same as the module of the minimal polynomials of the algebra $A_{2}$ over $P_{2}$, and a base for the latter module was given in Theorem 2 .

Now we turn to the general case, that is, either $n>2$ or $F \neq P_{2}$, or in case $n=2$ and $F=P_{2}$, some algebra $A^{(i)}$ is a total matric algebra of degree 2 over a field $\neq P_{2}$. For such algebras we prove the validity of Theorem 3, that is:

TheOREM 4. Let $A$ be a semi-simple algebra not of the types (I) or (II), then $f\left(x_{1}, \cdots, x_{m}\right)=0$ is a minimal identity of $A$ if and only if $m \geqq 2 n$, and

$$
f\left(x_{1}, \cdots, x_{m}\right)=\sum_{(i)} \alpha_{(i)} S\left(x_{i_{1}}, \cdots, x_{i_{2 n}}\right)
$$

where the sum ranges over all $C_{m, 2 n}$ combinations $(i)$ of $2 n$ letters out of $m$ letters, and $\alpha_{(i)}$ are in $F$.

Proof. We have already seen that $A$ satisfies an identity $f=0$ if and only if $f=0$ is satisfied by every constituent $A^{(i)}$. The minimal identities of the algebra $A^{(j)}$ of order $n_{j}^{2}=n^{2}$ over its centre are satisfied also by the algebra $A^{(i)}$ for which $n_{i} \leqq n_{j}=n$ if either $n>2$ or $F \neq P_{2}$, and when $n=2$ and $F=P_{2}$, the minimal identities of the algebra $A^{(j)}$ of order 4 over its centre $C$ such that $C \supset P_{2}$ are also satisfied by the other constituents $A^{(i)}$. This implies that $f$ is a minimal polynomial of $A$ if and only if $f$ is a minimal polynomial of that particular algebra $A^{(j)}$. Hence our theorem is an immediate consequence of Theorem 3 .

4. Algebras with radical. Let $r$ be the index of the radical $N$ of

${ }^{2}$ See also $[1, \S 4]$. 
the algebra $B$ over $F$, and let $n^{2}=\max \left(n_{1}^{2}, \cdots, n_{k}^{2}\right)$ where $n_{\mathfrak{\imath}}^{2}$ are the orders of the simple constituents of the difference algebra $B-N$. We prove:

Theorem 5. Denote by $m$ the degree of the minimal polynomial of $B$. Then:

(1) $2 n \leqq m \leqq 2 n r$;

(2) There exist algebras $B$ over $F$ with the index $r$ for which $m=2 n$, as well as algebras for which $m=2 n r$.

Proof. By [1, Theorem 9] we know that $B$ has identities of degree $2 n r$. This implies that $m \leqq 2 n r$. Since an identity satisfied by $B$ is satisfied also by $B-N$, it follows that $m \geqq 2 n$, that is, $2 n \leqq m \leqq 2 n r$.

Now let $B_{1}=A_{n}+N$ denote a direct sum of a total matric algebra $A_{n}$ over $F$ and a nilpotent algebra $N$ of index $t \leqq 2 n$. The identity $S\left(x_{1}, \cdots, x_{2 n}\right)=0$ is satisfied by both $A_{n}$ and $N$ and hence also by $B_{1}$. This implies that the minimum degree $m_{1}$ of $B_{1}$ is at most $2 n$. On the other hand $m_{1} \geqq 2 n$. It follows, therefore, that $m_{1}=2 n$.

Finally consider the algebra $B_{2}$, defined as the algebra of all matrices $\left(a_{i k}\right)$ of order $r$ over $F$ where $a_{i k}=0$ for $i>k$, that is, the ring of all matrices of order $r$ with zeros beneath the diagonal. The radical $N_{2}$ of this algebra is the set of all matrices $\left(a_{i k}\right)$ where $a_{i k}=0$ when $i \geqq k$, that is, $N_{2}$ is the set of all matrices of $B_{2}$ with zeros in the diagonal, and its index is therefore equal to $r$. The semi-simple algebra $B_{2}-N_{2}$ is a direct sum of $r$ commutative fields, that is, $n=1$. The minimum degree $m_{2}$ of $B_{2}$ is therefore subject to the inequality $2 \leqq m_{2} \leqq 2 r$. If $B_{2}$ has a polynomial identity of degree $m_{2}<2 r$, then evidently it possesses also an identity of degree $2 r-1$. Hence, by [2, Lemma 2], it follows that $B_{2}$ possesses also an identity $f\left(x_{1}, \cdots, x_{2 r-1}\right)=0$ of degree $2 r-1$, where $f$ is homogeneous and linear in all the indeterminates $x_{i}$. We may assume that the coefficient $\alpha$ of the monomial $x_{1} x_{2} \cdots x_{2 r-1}$ of $f$ is not zero.

Now substitute $x_{2 i-1}=e_{i i}, x_{2 i}=e_{i i+1}, i=1,2, \cdots, r-1, x_{2 r-1}=e_{r r}$. The only monomial of $f$ which yields under this substitution a nonzero element is $x_{1} \cdots x_{2 r-1}$, hence $f=\alpha e_{11} \neq 0$ which is a contradiction. This implies that $m_{2} \geqq 2 r$. Hence $m_{2}=2 r$, which completes the proof of the theorem.

\section{BIBLIOGRAPHY}

1. A. S. Amitsur and J. Levitzki, Minimal identities for algebras, Proceedings of the American Mathematical Society vol. 1 (1950) pp. 449-463.

2. I. Kaplansky, Rings and a polynomial identity, Bull. Amer. Math. Soc. vol. 54 (1948) pp. 575-580.

JERUSALEM UNIVERSITY 